\title{
Retrospective Cost Adaptive Control of Spacecraft Attitude Using Magnetic Actuators
}

\author{
Gerardo Cruz* and Dennis S. Bernstein ${ }^{\dagger}$ \\ University of Michigan, 1320 Beal Ave., Ann Arbor, MI 48109.
}

\begin{abstract}
We apply retrospective cost adaptive control (RCAC) to spacecraft attitude control with magnetic actuators. We compare two approaches to address the rank deficiency and time-varying nature of the input matrix. The first approach utilizes an average of the magnetic field based on a-priori knowledge, whereas the second approach uses three multi-input, single-output controllers. RCAC uses no information about the spacecraft inertia, and model information is limited to the input-output relation given by the first Markov parameter, which is computed from an inertia-free linearization of Euler's and Poisson's equations. We examine two problems for each of the controllers. For both problems, the spacecraft has an arbitrary initial angular rate and initial attitude. The objective for the first problem is to bring the spacecraft to rest at a specified attitude, while the second problem seeks to bring the spacecraft to spin about an inertially pointed body axis.
\end{abstract}




\section{Introduction}

The attitude of a spacecraft in low-Earth orbit can be either passively stabilized or actively controlled. Passive methods exploit environmental torques to achieve a stable attitude [1]. However, the range of reachable attitudes is limited by the specific method utilized. Active control actuators generate torques that provide three-axis control and are able to reach any attitude. However, the cost of actuators such as reaction wheels and thrusters for use in small spacecraft is high and their reliability is unproven. Alternatively, magnetic coils, produce torque by creating a magnetic field that interacts with the Earth's local magnetic field.

Magnetic coils are commonly used to reduce momentum in spacecraft that use momentum storage devices such as reaction wheels [2]. Magnetic actuators are also used to de-spin spacecraft after launch vehicle separation [3]. However, the torque produced by the coils is constrained to the plane orthogonal to the Earth's local magnetic field vector. This lack of instantaneous controllability along with low-torque capability, and low pointing accuracy make magnetic coils impractical for three-axis attitude control of large spacecraft. Yet, as the size of the spacecraft decreases and pointing accuracy requirements are relaxed, the benefits of magnetic coils, such as small size, ease of manufacturing, and low power consumption, outweigh the challenges in the design and operation of these control systems [4]. Thus,

the application of active magnetic coils for three-axis attitude control of small spacecraft has gained interest in recent years [5].

Attitude regulation methods for magnetic control typically rely on a model of the spacecraft dynamics and kinematics, the spacecraft mass properties, and a model of the magnetic field. Control techniques include proportionalderivative control, optimal control, and nonlinear methods [6]. However, these methods may fail when accurate modeling information is not available. Thus, a control law that reduces the required modeling information is desired.

In this paper we develop a controller that utilizes measurements of the local magnetic field without knowledge of the mass properties. This control law is based on retrospective cost adaptive control (RCAC) which is a multiinput, multi-output direct adaptive controller. RCAC utilizes the input and output history of the system combined with Markov parameters to update teh control law and determine the next control input [7], [8], [9], [10]. Previous applications of RCAC to spacecraft attitude control have been limited to thruster and reaction-wheel actuation [11], [12], [13]. In these applications, RCAC was applied to motion-to-rest (M2R) maneuvers, where the controller brings the body to rest at a specified attitude and motion-to-spin maneuvers (M2S), where the spacecraft is brought to spin about an inertially pointed body axis.

We apply RCAC to the attitude regulation of a rigid body spacecraft using magnetic actuators. First, we develop the nonlinear equations of motion for a rigid body spacecraft with magnetic actuators. Then, we describe the RCAC algorithm based on a linear time-invariant system. Next, we modify the RCAC formulation to accommodate the nonlinear equations, matrix-valued attitude state, and the rank deficiency of the input matrix. Finally, we present numerical results for M2R and M2S maneuvers for spacecraft with various inertia matrices in high-inclination orbits.

\section{Spacecraft Model}

We consider a rigid body controlled by magnetic torque actuators. The rotational motion is described by Euler's equation, and the kinematics are given by Poisson's equation. We define a body-fixed frame for the spacecraft, with the origin located at the center of mass, and we use an Earth-centered inertial (ECI) frame to determine the attitude of the spacecraft. Thus, the spacecraft motion is described by

$$
\begin{aligned}
J_{\mathrm{SC}} \dot{\omega} & =\left(J_{\mathrm{SC}} \omega\right) \times \omega+B_{\mathrm{SC}} u+z_{\mathrm{dist}}, \\
\dot{R} & =R \omega^{\times},
\end{aligned}
$$

where $\omega \in \mathbb{R}^{3}$ is the angular velocity of the body frame with respect to the ECI frame resolved in the spacecraft frame and $J_{\mathrm{SC}} \in \mathbb{R}^{3 \times 3}$ is the constant inertia dyadic of the spacecraft relative to the spacecraft center of mass resolved in the spacecraft frame. The proper orthogonal matrix (that is, the rotation matrix) $R \in \mathbb{R}^{3 \times 3}$ transforms the components of a vector resolved in the spacecraft frame into the components of the same vector resolved in the inertial frame, and $\omega^{\times}$is the skew-symmetric cross-product matrix of $\omega$.

The product $B_{\mathrm{SC}} u$, where $B_{\mathrm{SC}} \in \mathbb{R}^{3 \times l_{u}}$, determines the applied torque about each axis of the spacecraft frame due to the control input vector $u \in \mathbb{R}^{l_{u}}$. The vector $z_{d}$ represents disturbance torques, that is, all internal and external torques applied to the spacecraft aside from control torques. These disturbances may be due to onboard components, gravity gradients, solar pressure, atmospheric drag, or the ambient magnetic field. For convenience in (1) and (2) we omit the argument $t$, recognizing that $\omega, R, u$, and $z_{d}$ are time-varying quantities. 
We assume that both rate (inertial) and attitude (noninertial) measurements are available. Gyro measurements $y_{\text {rate }} \in \mathbb{R}^{3}$ provide measurements of the angular velocity resolved in the spacecraft frame, that is,

$$
y_{\text {rate }}=\omega .
$$

Attitude is measured indirectly using sensors such as star trackers. The attitude measurement is determined to be

$$
y_{\text {attitude }}=R \text {. }
$$

For simplicity, we assume that both rate and attitude measurements are available without noise and that gyro bias, if present, has been corrected.

The objective of the attitude control problem is to determine control inputs such that the spacecraft attitude $R$ follows a commanded attitude trajectory given by a possibly time-varying $\mathrm{C}^{1}$ rotation matrix $R_{\mathrm{d}}(t)$. For $t \geq 0, R_{\mathrm{d}}(t)$ is given by

$$
\begin{aligned}
\dot{R}_{\mathrm{d}}(t) & =R_{\mathrm{d}}(t) \omega_{\mathrm{d}}(t)^{\times}, \\
R_{\mathrm{d}}(0) & =R_{\mathrm{d} 0},
\end{aligned}
$$

where $\omega_{\mathrm{d}}$ is the desired, possibly time-varying angular velocity. The attitude error, that is, the rotation between $R(t)$ and $R_{\mathrm{d}}(t)$, is given by

$$
\tilde{R} \triangleq R_{\mathrm{d}}^{\mathrm{T}} R
$$

which satisfies Poisson's equation

$$
\dot{\tilde{R}}=\tilde{R} \tilde{\omega}^{\times}
$$

where the angular velocity error $\tilde{\omega}$ is defined by

$$
\tilde{\omega} \triangleq \omega-\tilde{R}^{\mathrm{T}} \omega_{\mathrm{d}}
$$

\section{The RCAC Algorithm}

RCAC is a discrete-time output-feedback controller that minimizes the command-following error corresponding to the performance variable $z$. The algorithm does not require detailed plant information, instead, RCAC uses knowledge of the system's input response as described by Markov parameters. Although RCAC is derived for linear systems, we apply it to the nonlinear spacecraft model by using Markov parameters from the linearized dynamics.

\section{III.A. The Extended System and Retrospective Cost}

Consider the MIMO discrete-time linear system

$$
\begin{aligned}
x(k+1) & =A x(k)+B u(k), \\
z(k) & =E_{1} x(k)-E_{0} r(k),
\end{aligned}
$$

where $x(k) \in \mathbb{R}^{l_{x}}, z(k) \in \mathbb{R}^{l_{z}}, u(k) \in \mathbb{R}^{l_{u}}, r(k) \in \mathbb{R}^{l_{r}}$, and $k \geq 0$. We can rewrite (11) as

$$
z(k)=E_{1} A x(k-1)+E_{1} B u(k-1)-E_{0} r(k) .
$$

Then, we collect the terms in (12) such that

$$
z(k)=S(k)+\mathcal{H} u(k-1)
$$

where

$$
S(k)=E_{1} A x(k-1)-E_{0} r(k)
$$

and $\mathcal{H}=E_{1} B$ is the first Markov parameter of the system. 
For a positive integer $s$, define the extended performance as

$$
\begin{aligned}
\bar{Z}(k) & =\left[\begin{array}{c}
z(k) \\
z(k-1) \\
\vdots \\
z(k-s)
\end{array}\right] \\
& =\bar{S}(k)+\bar{H} \bar{U}(k-1),
\end{aligned}
$$

where

$$
\begin{aligned}
\bar{S}(k) & =\left[\begin{array}{c}
S(k) \\
S(k-1) \\
\vdots \\
S(k-s)
\end{array}\right] \in \mathbb{R}^{s l_{z}}, \\
\bar{H} & =\left[\begin{array}{ccc}
\mathcal{H} & & \\
\mathcal{H} & & \\
& \ddots & \\
& & \mathcal{H}
\end{array}\right] \in \mathbb{R}^{s l_{z} \times s l_{u}}, \\
\bar{U}(k-1) & =\left[\begin{array}{c}
u(k-1) \\
u(k-2) \\
\vdots \\
u(k-s)
\end{array}\right] \in \mathbb{R}^{s l_{u}} .
\end{aligned}
$$

We replace the control inputs in (15) with the retrospective controls $\hat{\bar{U}}(k-1)$ and define the extended retrospective performance

$$
\hat{\bar{Z}}(k)=\bar{S}(k)+\bar{H} \hat{\bar{U}}(k-1) .
$$

Next, we subtract the extended performance in (15) from the extended retrospective performance in (19) and obtain

$$
\hat{Z}(k)=Z(k)-H \bar{U}(k-1)+\hat{\bar{U}}(k-1) .
$$

We wish to find the retrospective control inputs $\hat{\bar{U}}(k-1)$ that minimize the retrospective performance $\hat{\bar{Z}}(k)$.

Thus, we define the retrospective cost function

$$
J(U(k-1), k) \triangleq \hat{\bar{Z}}^{\mathrm{T}}(k) R_{Z}(k) \hat{\bar{Z}}(k)+\hat{\bar{U}}(k-1)^{\mathrm{T}} R_{U}(k) \hat{\bar{U}}(k-1) Z(k),
$$

where $R_{Z}(k) \in \mathbb{R}^{s l_{z} \times s l_{z}}$ and $R_{U}(k) \in \mathbb{R}^{s l_{u} \times s l_{u}}$ are positive-definite weighting matrices on the performance and the control respectively. Using (20) we rewrite the cost function as

$$
J(U(k-1), k)=\hat{\bar{U}}(k-1)^{\mathrm{T}} \mathcal{A}(k) \hat{\bar{U}}(k-1)+\hat{\bar{U}}^{\mathrm{T}}(k-1) \mathcal{B}^{\mathrm{T}}(k) \hat{\bar{U}}+\mathcal{C}(k),
$$

where

$$
\begin{aligned}
& \mathcal{A}(k) \triangleq \bar{H}^{\mathrm{T}} R_{Z}(k) \bar{H}+R_{U}(k), \\
& \mathcal{B}(k) \triangleq 2 \bar{H}^{\mathrm{T}} R_{Z}(k)[\bar{Z}(k)-\bar{H} \bar{U}(k-1)], \\
& \mathcal{C}(k) \triangleq \bar{Z}^{\mathrm{T}}(k) R_{Z}(k) \bar{Z}(k)-2 \bar{Z}^{\mathrm{T}}(k) R_{Z}(k) \bar{H} \bar{U}(k-1)+\bar{U}^{\mathrm{T}}(k-1) \bar{H}^{\mathrm{T}} R_{Z}(k) \bar{H} \bar{U}(k-1) .
\end{aligned}
$$

If $\mathcal{A}(k)$ is positive definite, the unique minimizer for $J(\bar{U}(k-1), k)$ is

$$
\hat{\bar{U}}(k-1)=-\frac{1}{2} \mathcal{A}^{-1}(k) \mathcal{B}(k) .
$$




\section{III.B. Controller Construction}

We utilize the retrospective controls $\hat{\bar{U}}(k-1)$ in order to compute the next control input $u(k)$. We design a strictly proper time-series controller of order $n_{\mathrm{c}}$ given by

$$
u(k)=\sum_{i=1}^{n_{\mathrm{c}}} M_{i}(k) u(k-i)+\sum_{i=1}^{n_{\mathrm{c}}} N_{i}(k) z(k-i),
$$

where, for all $i=1, \ldots, n_{\mathrm{c}}, M_{i}(k) \in \mathbb{R}^{l_{u} \times l_{u}}$ and $N_{i}(k) \in \mathbb{R}^{l_{u} \times l_{z}}$. The control (27) can be expressed as

$$
u(k)=\theta(k) \phi(k-1),
$$

where

$$
\theta(k) \triangleq\left[M_{1}(k) \cdots M_{n_{\mathrm{c}}}(k) N_{1}(k) \cdots N_{n_{\mathrm{c}}}(k)\right] \in \mathbb{R}^{l_{u} \times n_{\mathrm{c}}\left(l_{u}+l_{z}\right)},
$$

and

$$
\phi(k-1) \triangleq\left[\begin{array}{c}
u(k-1) \\
\vdots \\
u\left(k-n_{\mathrm{c}}\right) \\
z(k-1) \\
\vdots \\
z\left(k-n_{\mathrm{c}}\right)
\end{array}\right] \in \mathbb{R}^{n_{\mathrm{c}}\left(l_{u}+l_{z}\right)}
$$

\section{III.C. Recursive Least Squares Update of $\theta(k)$}

We compute the parameter $\theta(k)$ by solving a recursive least squares problem. In order for the applied controls $\bar{U}(k)$ to approach the retrospective controls $\hat{\bar{U}}(k)$ we minimize the cost function

$$
\begin{aligned}
J(\theta(k)) & \triangleq[\hat{u}(k)-u(k)]^{\mathrm{T}}[\hat{u}(k)-u(k)], \\
& =[\hat{u}(k)-\theta(k) \phi(k-1)]^{\mathrm{T}}[\hat{u}(k)-\theta(k) \phi(k-1)], \\
& =\|\hat{u}(k)-\theta(k) \phi(k-1)\|^{2} .
\end{aligned}
$$

The minimizer for (31) is

$$
\begin{aligned}
\theta^{\mathrm{T}}(k) \triangleq & \theta^{\mathrm{T}}(k-1)+P(k-1) \phi\left(k-q_{g}-1\right)\left[\phi^{\mathrm{T}}\left(k-q_{g}-1\right) P(k-1) \phi\left(k-q_{g}-1\right)\right]^{-1} \\
& \cdot\left[\theta(k-1) \phi\left(k-q_{g}-1\right)-\hat{u}\left(k-q_{g}\right)\right]^{\mathrm{T}} .
\end{aligned}
$$

The error covariance is updated by

$$
\begin{aligned}
P(k) \triangleq & P(k-1)-P(k-1) \phi\left(k-q_{g}-1\right) \\
& \cdot\left[\phi^{\mathrm{T}}\left(k-q_{g}-1\right) P(k-1) \phi\left(k-q_{g}-1\right)\right]^{-1} \phi^{\mathrm{T}}\left(k-q_{g}-1\right) P(k-1) .
\end{aligned}
$$

\section{Modifications to RCAC for Magnetic Control of Spacecraft Attitude}

RCAC is applied to discrete-time linear systems where the state $x(k)$ in (10) is a vector. However, the spacecraft equations (1), (2) are nonlinear, and the attitude state $R$ is a matrix. Thus, the spacecraft equations must be modified in order to apply RCAC as developed in SectionIII Furthermore, the magnetic constraints on the control torque introduce additional difficulties in the computation of the retrospective controls $\hat{\bar{U}}(k-1)$ in (26) and the implementation of the control input $u(k)$ in (28).

Thus, we develop the equations for a vector performance variable $z$ and obtain a suitable matrix $\mathcal{H}$ for the nonlinear spacecraft equations based on the linearized system. Then, we present a torque-allocation scheme to transform the torques computed by RCAC into magnetic dipoles. Finally, we develop two methods for managing the rank deficiency in $\mathcal{H}$ caused by the singular input matrix. 


\section{IV.A. Performance Variable for Attitude Control}

We express the rotation matrix $\tilde{R}$ as

$$
\tilde{R}=\left[\begin{array}{c}
\tilde{r}_{1} \\
\tilde{r}_{2} \\
\tilde{r}_{3}
\end{array}\right]
$$

where, for $i=1,2,3, \tilde{r}_{i} \in \mathbb{R}^{1 \times 3}$ is a row of $\tilde{R}$. As in [11], we define the vector state

$$
\tilde{r} \triangleq\left[\begin{array}{lll}
\tilde{r}_{1} & \tilde{r}_{2} & \tilde{r}_{3}
\end{array}\right]^{\mathrm{T}}
$$

Next, we utilize the attitude error vector in [14] given by

$$
S \triangleq \sum_{1}^{3} a_{i}\left(\tilde{R}^{\mathrm{T}} e_{i}\right) \times e_{i}=-M_{a} R_{a} \tilde{r},
$$

where the constants $a_{i}$ are positive and distinct, the vector $e_{i} \in \mathbb{R}^{3}$ for $i=1,2,3$ is the $i$ th column of the $3 \times 3$ identity matrix, and

$$
\begin{aligned}
& M_{a}=\left[\begin{array}{lll}
e_{1}^{\times} & e_{2}^{\times} & e_{3}^{\times}
\end{array}\right], \in \mathbb{R}^{3 \times 9} \\
& R_{a}=\left[\begin{array}{lll}
a_{1} I_{3} & & \\
& a_{2} I_{3} & \\
& & a_{3} I_{3}
\end{array}\right] \in \mathbb{R}^{9 \times 9} .
\end{aligned}
$$

Including the angular velocity error in (9) yields the performance variable

$$
z=\left[\begin{array}{c}
\tilde{\omega} \\
S
\end{array}\right]
$$

\section{IV.B. Markov parameter}

We rewrite (1) and (2) using the errors $\tilde{r}$ and $\tilde{\omega}$ such that

$$
\dot{\tilde{\omega}}=J_{\mathrm{SC}}^{-1}\left[\left[J_{\mathrm{SC}}\left(\tilde{\omega}+\mathcal{D}\left(\omega_{d}\right) r\right)\right] \times\left(\tilde{\omega}+\mathcal{D}\left(\omega_{d}\right) r\right)\right]+\tilde{\omega} \times\left[\mathcal{D}\left(\omega_{d}\right) r\right]-\mathcal{D}\left(\dot{\omega}_{d}\right) r+J_{\mathrm{SC}}^{-1}\left(B_{\mathrm{SC}} u+z_{d}\right),
$$

where, for $x \in \mathbb{R}^{3}$ with components $x_{1}, x_{2}, x_{3}$,

$$
\mathcal{D}(x) \triangleq\left[\begin{array}{lll}
x_{1} I_{3} & x_{2} I_{3} & x_{3} I_{3}
\end{array}\right]
$$

and

$$
\dot{\tilde{r}}=\left[\begin{array}{lll}
-\tilde{\omega}^{\times} & & \\
& -\tilde{\omega}^{\times} & \\
& & -\tilde{\omega}^{\times}
\end{array}\right] \tilde{r} .
$$

We compute the discrete-time system matrices $A, B, E_{1}$ by linearizing (40) and (42) about $\tilde{\omega}_{e}=0$ and $\tilde{R}=I_{3}$ followed by discretization with the controller time step $h$. Linearization yields the continuous-time dynamics matrices

$$
\begin{aligned}
A_{c} & =\left[\begin{array}{cc}
0_{3 \times 3} & 0_{3 \times 9} \\
-M_{a}^{\mathrm{T}} & 0_{9 \times 9}
\end{array}\right], \\
B_{c} & =\left[\begin{array}{c}
J_{\mathrm{SC}}^{-1} B_{\mathrm{SC}} \\
0_{9 \times 3}
\end{array}\right], \\
C_{c} & =\left[\begin{array}{cc}
I_{3} & 0_{3 \times 9} \\
0_{3 \times 3} & -M_{a} R_{a}
\end{array}\right] .
\end{aligned}
$$


Discretization of $A_{c}, B_{c}, C_{c}$ with the controller time step $h$ yields

$$
\begin{aligned}
A & =e^{A_{c} h}=\left[\begin{array}{cc}
I_{3} & 0_{3 \times 9} \\
-h M_{a}^{\mathrm{T}} & I_{9}
\end{array}\right], \\
B & =\left(\int_{0}^{h} A d \tau\right) B_{c}=\left[\begin{array}{c}
h J_{\mathrm{SC}}^{-1} B_{\mathrm{SC}} \\
-\frac{h^{2}}{2} M_{a}^{\mathrm{T}} J_{\mathrm{SC}}^{-1} B_{\mathrm{SC}}
\end{array}\right], \\
E_{1} & =C_{c} .
\end{aligned}
$$

The Markov parameter corresponding to the performance in (39) is given by

$$
\mathcal{H}=E_{1} B=\left[\begin{array}{c}
h J_{\mathrm{SC}}^{-1} B_{\mathrm{SC}} \\
\frac{1}{2} h^{2} M_{a} R_{a} M_{a}^{\mathrm{T}} J_{\mathrm{SC}}^{-1} B_{\mathrm{SC}}
\end{array}\right] .
$$

As in [11], we remove the inertia information from $\mathcal{H}$ and define

$$
H \triangleq \alpha\left[\begin{array}{c}
h B_{\mathrm{SC}} \\
\frac{1}{2} h^{2} M_{a} R_{a} M_{a}^{\mathrm{T}} B_{\mathrm{SC}}
\end{array}\right],
$$

where $\alpha$ is a positive scalar.

\section{IV.C. Magnetic Dipole Allocation}

The command $u$ computed by RCAC is the desired torque. However, the magnetic coils must generate these torques using a magnetic dipole command $d$. Given the control torque $u$ commanded by RCAC we must compute the required dipole $d$. The resulting dipole creates a torque vector that is orthogonal to the local, time-varying magnetic field $b(t) \in \mathbb{R}^{3}$.

The torque obtained from a magnetic dipole $d(t)$ and the Earth's magnetic field $b(t)$ is given by

$$
\tau(t)=-b(t)^{\times} d(t) .
$$

We replace $\tau$ in (51) with the desired control torque $u$, and solve formally for $d$ by using the generalized inverse of the skew-symmetric matrix $b(t)^{\times}$,

$$
b(t)^{\times+}=-\frac{b(t)^{\times}}{b(t)^{\mathrm{T}} b(t)}
$$

and obtain

$$
d(t)=-b(t)^{\times+} u=\frac{b(t)^{\times}}{b(t)^{\mathrm{T}} b(t)} u .
$$

The generalized inverse projects the desired torque onto the plane orthogonal to $b(t)$ and allocates the necessary dipole $d(t)$. Thus, the control torque applied to the spacecraft is

$$
\tau(t)=-b(t)^{\times} d(t)=B_{\mathrm{SC}}(t) u,
$$

where

$$
B_{\mathrm{SC}}(t) \triangleq \frac{-b(t)^{\times} b(t)^{\times}}{b(t)^{\mathrm{T}} b(t)} .
$$

Note that, at each time instant, the rank of $B_{\mathrm{SC}}(t)$ is 2 . 


\section{Rank deficiency of $B_{\mathrm{SC}}$}

In previous approaches to spacecraft attitude control [11], [12], [13], RCAC was set up as a multi-input, multioutput controller. The input matrix $B_{\mathrm{SC}}(t)$ in (1) is used to compute the Markov parameter matrix $\bar{H}$ in (17). However, since $b(t)^{\times}$is skew symmetric, $\bar{H}$ is rank deficient, which prevents the inversion of $\mathcal{A}$ in (26) in the absence of the control weighting matrix $R_{U}$. Although it is possible to create a full rank $\mathcal{A}$ by using the control weighting matrix $R_{U}$, numerical studies suggest that this does not result in a successful control law.

Thus, to ensure that the product $\bar{H}^{\mathrm{T}} R_{Z} \bar{H}$ in (23) is invertible, we propose two modifications to the previous attitude control RCAC implementations. The first approach utilizes the average of the input matrix $B_{\mathrm{SC}}(t)$. This average matrix is shown in [15] to have full-rank for orbits that are non-equatorial, thus the resulting Markov parameter is left invertible. The second approach uses an alternate control architecture including three separate multi-input, single-output RCAC controllers instead of one multi-input, multi-output controller.

\section{V.A. Averaged Markov parameter}

Define the input matrix $B_{\mathrm{SC}}(t)$ resolved in the ECI frame as

$$
\left.B_{\mathrm{SC}}(t)\right|_{\mathrm{ECI}}=B_{\mathrm{SC}}^{\prime}(t) \triangleq \frac{-b^{\prime}(t)^{\times} b^{\prime}(t)^{\times}}{b^{\prime}(t)^{\mathrm{T}} b^{\prime}(t)}
$$

where $b^{\prime}(t)$ is the magnetic field vector resolved in the ECI frame. Next, we compute the average of $B_{\mathrm{SC}}^{\prime}(t)$ over several orbits

$$
\tilde{B}_{\mathrm{SC}}^{\prime}=\lim _{T \rightarrow \infty} \frac{1}{T} \int_{0}^{T} B_{\mathrm{SC}}^{\prime}(t) d t .
$$

The averaged input matrix (57) is used to prove controllability and stability in [15]. We use this approach to obtain a full rank Markov parameter for RCAC. First, we transform the averaged input matrix into the spacecraft body frame

$$
\left.\tilde{B}_{\mathrm{SC}} \triangleq \tilde{B}_{\mathrm{SC}}^{\prime}\right|_{B}=R^{\mathrm{T}} \tilde{B}_{\mathrm{SC}}^{\prime} R .
$$

Then, using the average matrix in resolved in the body frame we construct the average Markov parameter

$$
\tilde{\mathcal{H}}=\alpha\left[\begin{array}{c}
h J_{\mathrm{SC}}^{-1} \tilde{B}_{\mathrm{SC}} \\
\frac{1}{2} h^{2} M_{a} R_{a} M_{a}^{\mathrm{T}} J_{\mathrm{SC}}^{-1} \tilde{B}_{\mathrm{SC}}
\end{array}\right] .
$$

As in (50), we remove the inertia and obtain,

$$
\tilde{H}=\alpha\left[\begin{array}{c}
h \tilde{B}_{\mathrm{SC}} \\
\frac{1}{2} h^{2} M_{a} R_{a} M_{a}^{\mathrm{T}} \tilde{B}_{\mathrm{SC}}
\end{array}\right],
$$

which is left invertible for non-equatorial orbits.

\section{V.B. Decentralized RCAC}

For the second method, we synthesize the desired torque $u$ using three independent multi-input single-output RCAC control loops. A similar architecture is used in [16] for angular velocity control using a heuristic approach to controller construction. We define the performance for each RCAC block as

$$
z_{i}(k) \triangleq\left[\begin{array}{c}
\tilde{\omega}_{i} \\
S_{i}
\end{array}\right]=C_{i}^{\prime} z(k),
$$

where $S_{i}$ and $\tilde{\omega}_{i}$ are the $i$ th components of $S$ and $\tilde{\omega}$, respectively, and

$$
C_{i}^{\prime} \triangleq\left[\begin{array}{cc}
e_{i}^{\mathrm{T}} & 0_{1 \times 3} \\
0_{1 \times 3} & e_{i}^{\mathrm{T}}
\end{array}\right] .
$$


We rewrite (61) using (10) and obtain

$$
z_{i}(k)=C_{i}^{\prime} E_{1} A x(k-1)+\sum_{j=1}^{3} C_{i}^{\prime} E_{1} B e_{j} u_{j}(k-1),
$$

where $u_{j}(k)$ is the $j$ th component of $u(k)$.

To compute the Markov parameter we assume that the sensor and actuator frames are aligned and that each component of the performance $z(k)$ is only affected by the corresponding component of the control $u(k-1)$ such that

$$
z_{i}(k) \approx C_{i}^{\prime} E_{1} A x(k-1)+C_{i}^{\prime} E_{1} B e_{i} u_{i}(k-1) .
$$

Thus, the Markov parameter for the $i$ th multi-input, single-output RCAC is

$$
\begin{aligned}
\mathcal{H}_{i}(t) & =C_{i}^{\prime} E_{1} B e_{i} \\
& =\left[\begin{array}{c}
h e_{i}^{\mathrm{T}} J_{\mathrm{SC}}^{-1} B_{\mathrm{SC}}(t) \\
\frac{1}{2} h^{2} e_{i}^{\mathrm{T}} M_{a} R_{a} M_{a}^{\mathrm{T}} J_{\mathrm{SC}}^{-1} B_{\mathrm{SC}}(t)
\end{array}\right] e_{i} .
\end{aligned}
$$

Removing the inertia yields

$$
H_{i}^{\prime}(t) \triangleq\left[\begin{array}{c}
h e_{i}^{\mathrm{T}} B_{\mathrm{SC}}(t) \\
\frac{1}{2} h^{2} e_{i}^{\mathrm{T}} M_{a} R_{a} M_{a}^{\mathrm{T}} B_{\mathrm{SC}}(t)
\end{array}\right] e_{i},
$$

which is left invertible. This approach ignores the coupling between axes and only requires knowledge of the alignment between actuators and sensors.

\section{Numerical Examples}

\begin{tabular}{|l|c|}
\hline Parameter & Value \\
\hline Inclination & $87^{\circ}$ \\
\hline Radius & $450[\mathrm{~km}]$ \\
\hline Right ascension of ascending node & 0 \\
\hline Argument of perigee & 0 \\
\hline Mean anomaly & 0 \\
\hline Period $\left(T_{\text {orbit }}\right)$ & $5615[\mathrm{sec}]$ \\
\hline \multicolumn{2}{|c|}{ Table 1. Orbital parameters. } \\
\hline
\end{tabular}

\begin{tabular}{|l|c|}
\hline parameter & Value \\
\hline$n_{c}$ & 10 \\
\hline$P_{0}$ & $100 I$ \\
\hline$R_{U}$ & $10 I$ \\
\hline$s$ & $\frac{T_{\text {orbit }}}{h}$ \\
\hline$\theta_{0}$ & 0 \\
\hline$a_{1}$ & 1 \\
\hline$a_{2}$ & 2 \\
\hline$a_{3}$ & 3 \\
\hline$h$ & 10 [sec] \\
\hline$\alpha$ & 0.1 \\
\hline
\end{tabular}

Table 2. RCAC parameters.

Consider a rigid spacecraft around a high-inclination circular orbit given by the orbital parameters in Table 1 . The magnetic field is computed using the International Geomagnetic Reference Field (IGRF) model [17]. Furthermore, 
all disturbances $z_{\mathrm{d}}$ are assumed to be zero. The RCAC parameters used are shown in Table 2. Furthermore, the performance weighting is given by

$$
R_{Z}=\left[\begin{array}{cc}
\epsilon I_{3} & 0 \\
0 & \epsilon^{2} I_{3}
\end{array}\right]
$$

where $\epsilon$ is a positive number. The scaling requirements between the angular velocity and attitude error terms is explained in [15]. For the M2R examples, we set $\epsilon=10^{-5}$.

\section{VI.A. M2R Examples}

Let the initial motion of the spacecraft be described by

$$
\omega(0)=0.001\left[\begin{array}{lll}
0 & -1 & 0
\end{array}\right]^{\mathrm{T}} \mathrm{rad} / \mathrm{sec} .
$$

We describe the initial and desired attitudes using eigenaxis rotations as defined by Rodrigues' equation

$$
\mathcal{R}\left(\theta_{e}, \hat{n}_{e}\right)=\cos \left(\theta_{e}\right) I_{3}+\left(1-\cos \left(\theta_{e}\right)\right) n_{e} n_{e}^{\mathrm{T}}+\sin \left(\theta_{e}\right) n_{e}^{\times},
$$

where $\theta_{e}$ is the eigenangle and $\hat{n}_{e} \in \mathbb{R}^{3}$ is the eigenaxis. Thus, the initial attitude be given by an eigenaxis rotation of $\theta_{0}=90^{\circ}$ about the vector

$$
n_{0}=\left[\begin{array}{lll}
-0.03 & -0.9 & 0.03
\end{array}\right]^{\mathrm{T}} .
$$

The goal of the controller is to bring the spacecraft to rest, that is, $\omega_{d}=0$, at the inertial attitude given by the eigenaxis rotation of $\theta_{d}=96^{\circ}$ about the vector

$$
n_{d}=\left[\begin{array}{lll}
1 & 1 & -1
\end{array}\right]^{\mathrm{T}} .
$$

We test the M2R maneuver on three different rigid bodies, namely, a sphere, a cylinder, and an arbitrary body.

We assume that the sensor and actuator axes are aligned such that the inertia matrices resolved in the body frame are given by

$$
\begin{aligned}
J_{\text {sphere }} & =\operatorname{diag}(10,10,10) \mathrm{kg}-\mathrm{m}^{2}, \\
J_{\text {cylinder }} & =\operatorname{diag}(10,10,5) \mathrm{kg}-\mathrm{m}^{2}, \\
J_{\text {arbitrary }} & =\left[\begin{array}{ccc}
5 & -0.1 & -0.5 \\
-0.1 & 2 & 1 \\
-0.5 & 1 & 3.5
\end{array}\right] \mathrm{kg}-\mathrm{m}^{2} .
\end{aligned}
$$

We compare the performance of both approaches to magnetic control for the M2R maneuver using the inertias in (73), (74), and (75). Figure 1 shows the results for the sphere inertia, Figure 2 shows the results for the cylinder inertia, and Figure 3 shows the results for the arbitrary inertia. The dipoles shown in the examples indicate that both implementations, averaged and decentralized, command dipoles of the same magnitude given the same tuning parameters in Table 2. Thus, we compare both approaches based on settling time of the eigenaxis attitude error $\theta_{\text {eig. }}$. For all three inertias, the centralized approach based on the average Markov parameter $\tilde{H}$ settles faster than the decentralized version based on the Markov parameter $H_{i}^{\prime}$. 


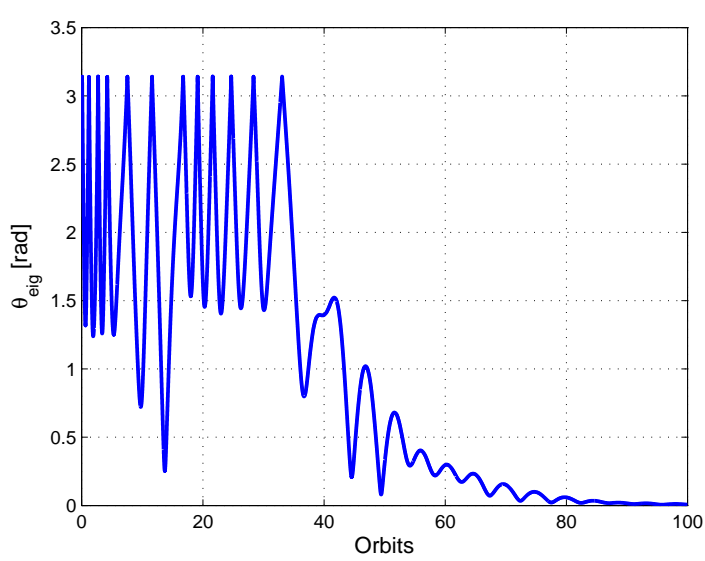

(a) Eigenaxis attitude error for MIMO RCAC

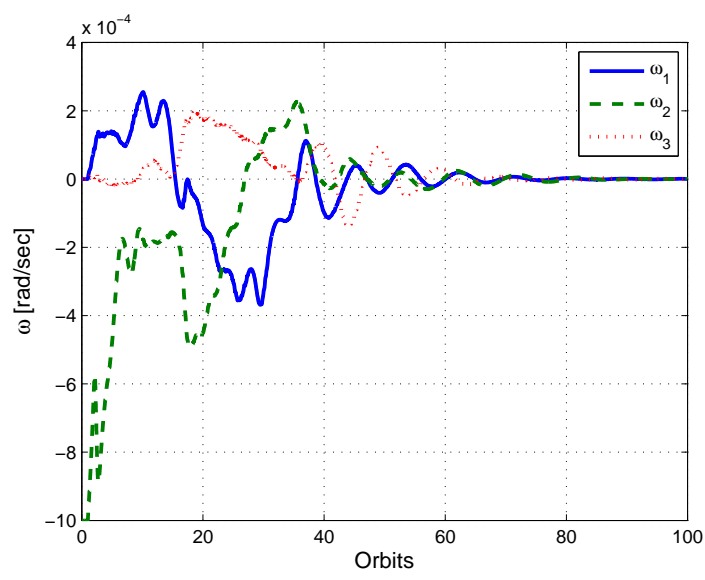

(c) Angular velocity for MIMO RCAC

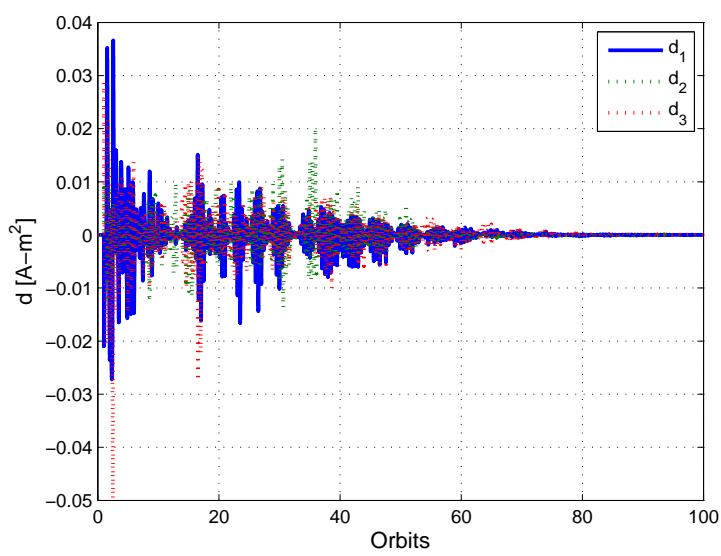

(e) Commanded Dipole for MIMO RCAC

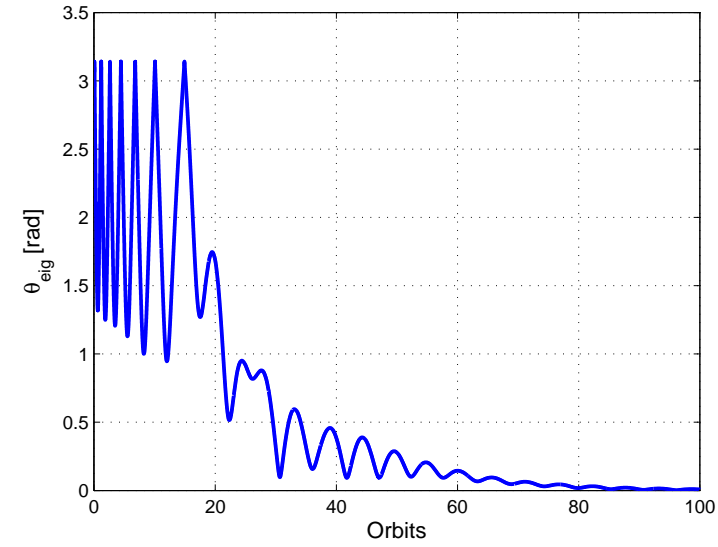

(b) Eigenaxis attitude error for decentralized RCAC

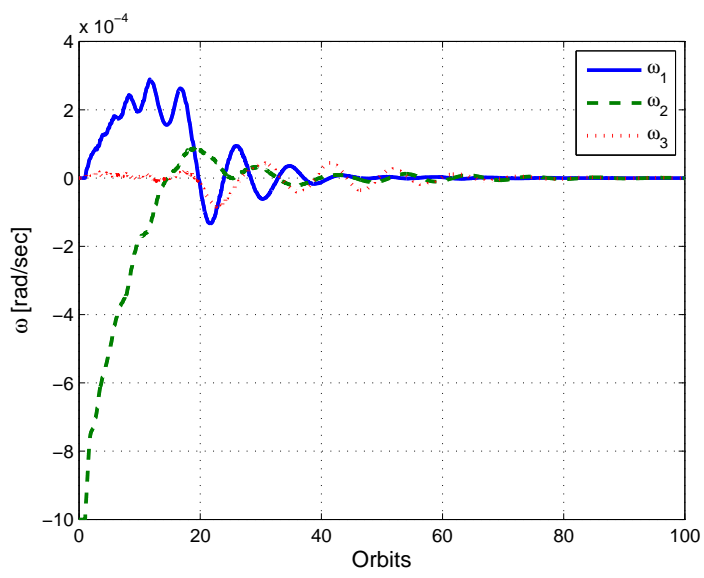

(d) Angular velocity for decentralized RCAC

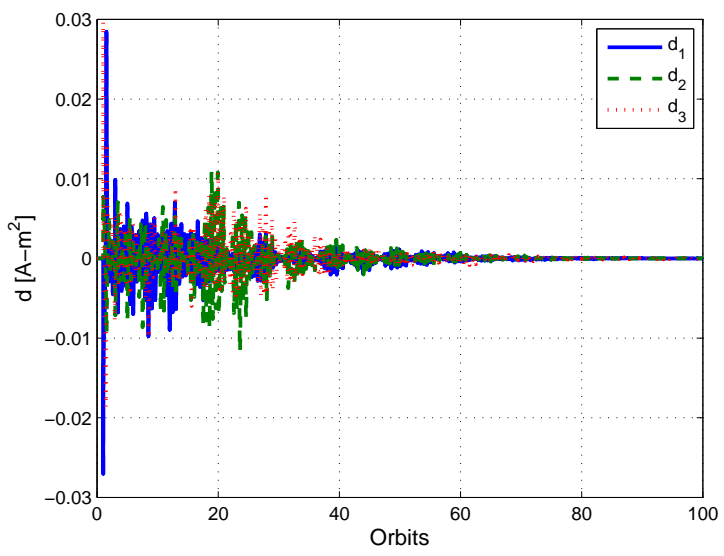

(f) Commanded Dipole for MIMO RCAC

Figure 1. Comparison of RCAC using the average Markov parameter $\tilde{H}$ versus the decentralized approach for the M2R maneuver for the sphere inertia $J_{\text {sphere }}$ in (73). 


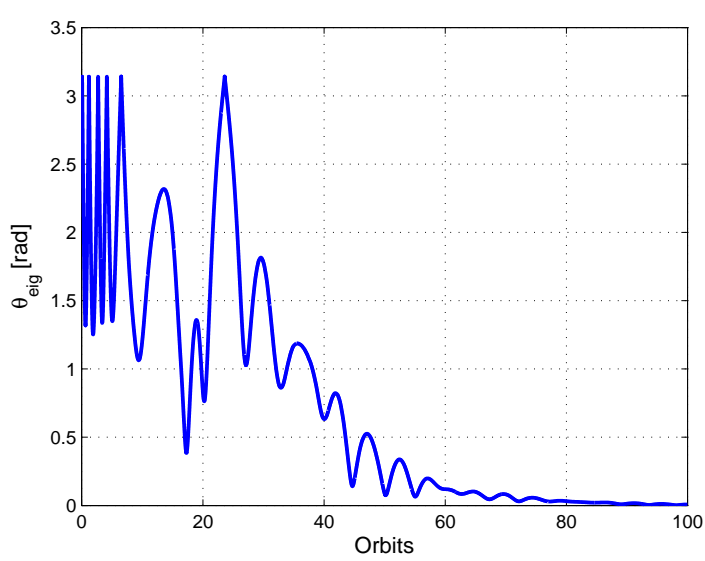

(a) Eigenaxis attitude error for MIMO RCAC

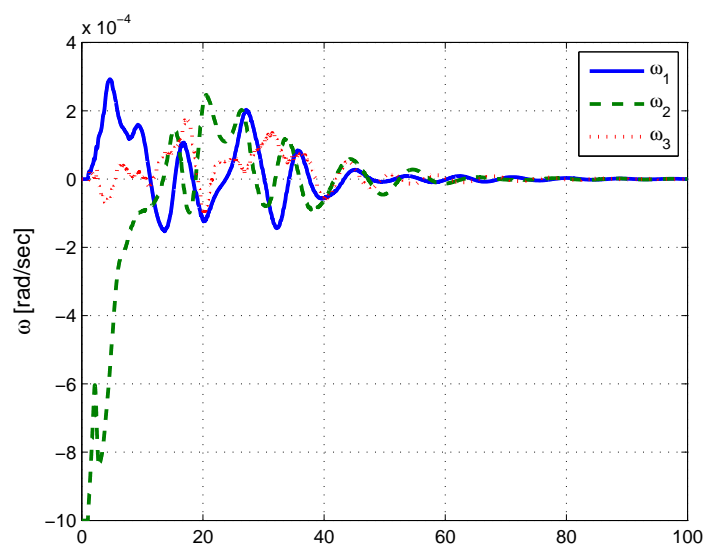

(c) Angular velocity for MIMO RCAC

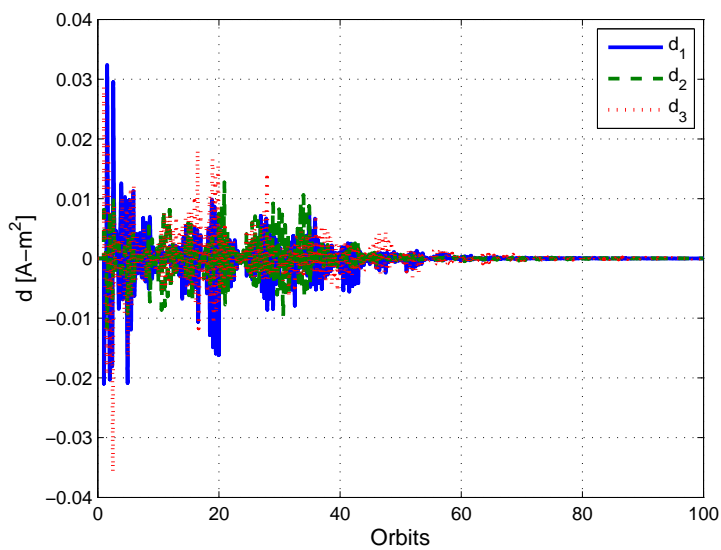

(e) Commanded Dipole for MIMO RCAC

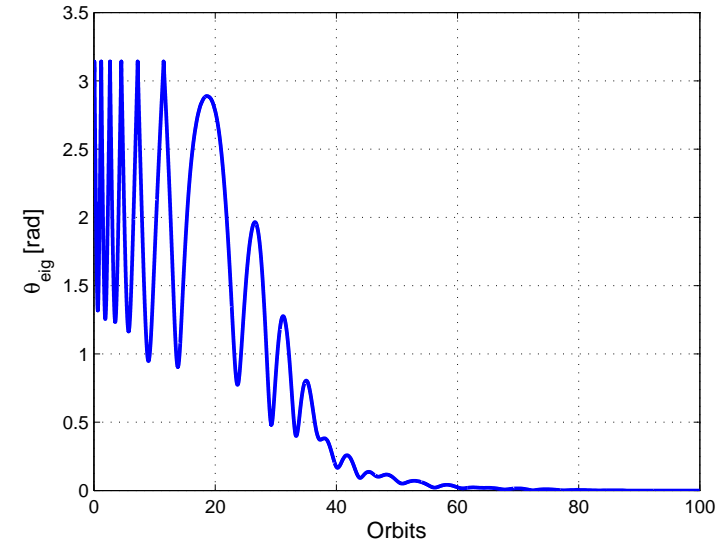

(b) Eigenaxis attitude error for decentralized RCAC

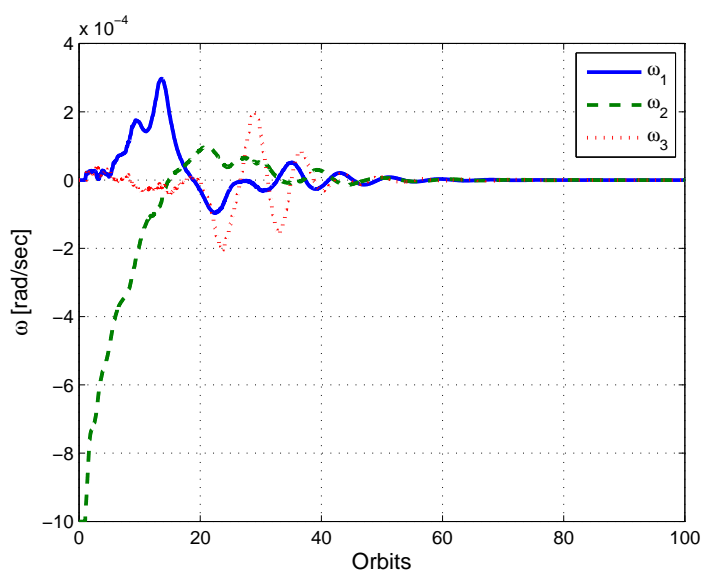

(d) Angular velocity for decentralized RCAC

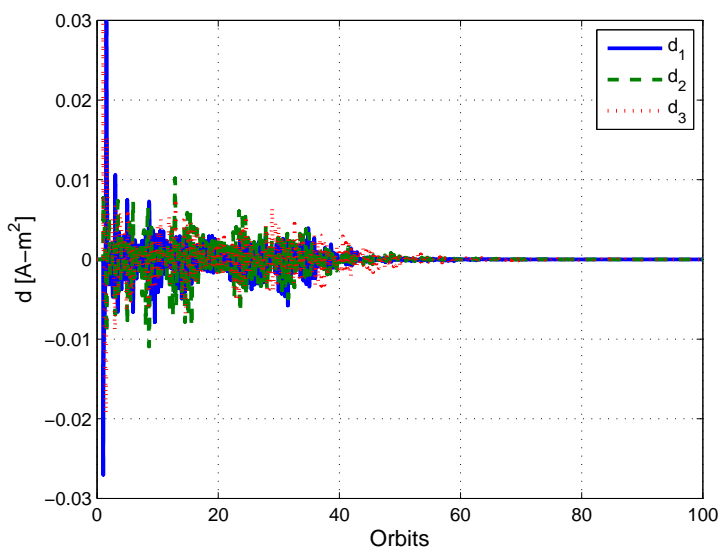

(f) Commanded Dipole for MIMO RCAC

Figure 2. Comparison of RCAC using the average Markov parameter $\tilde{H}$ versus the decentralized approach for the M2R maneuver for the cylinder inertia

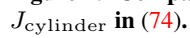




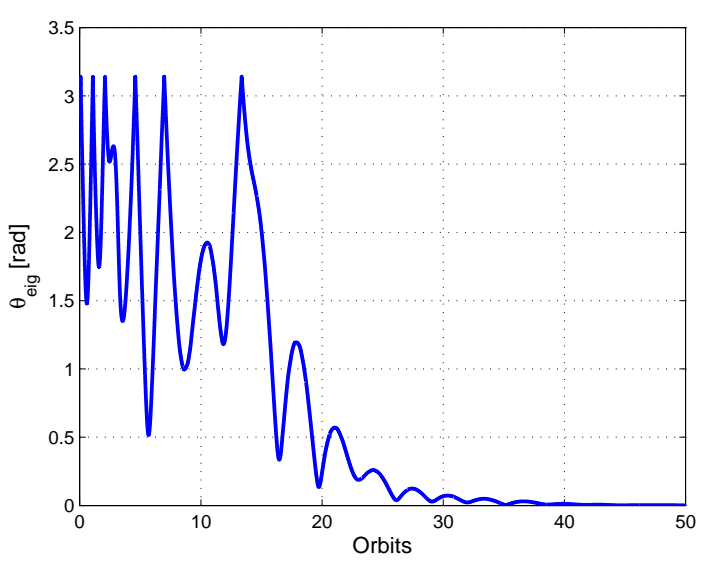

(a) Eigenaxis attitude error for MIMO RCAC

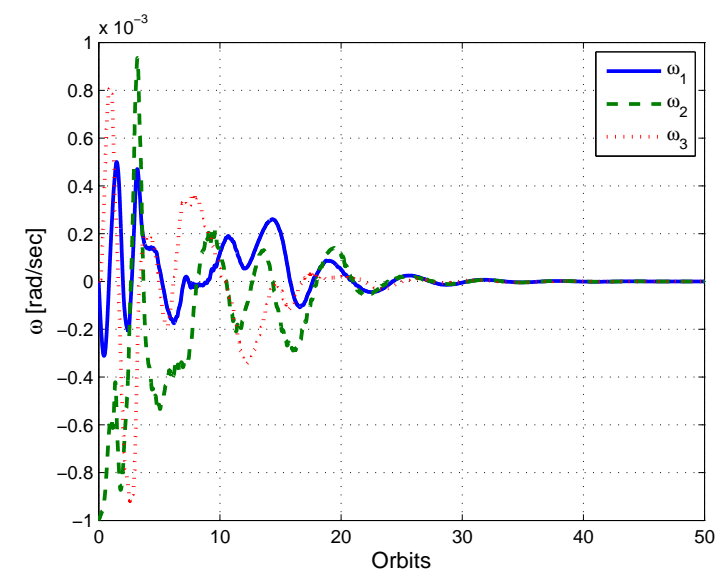

(c) Angular velocity for MIMO RCAC

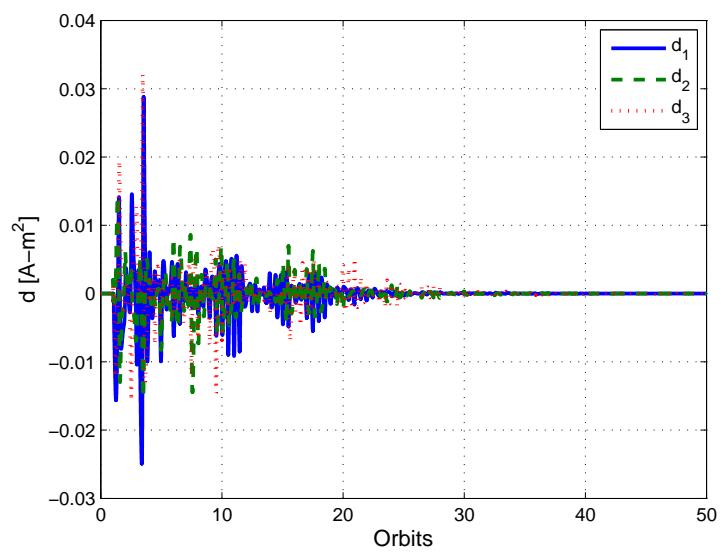

(e) Commanded Dipole for MIMO RCAC

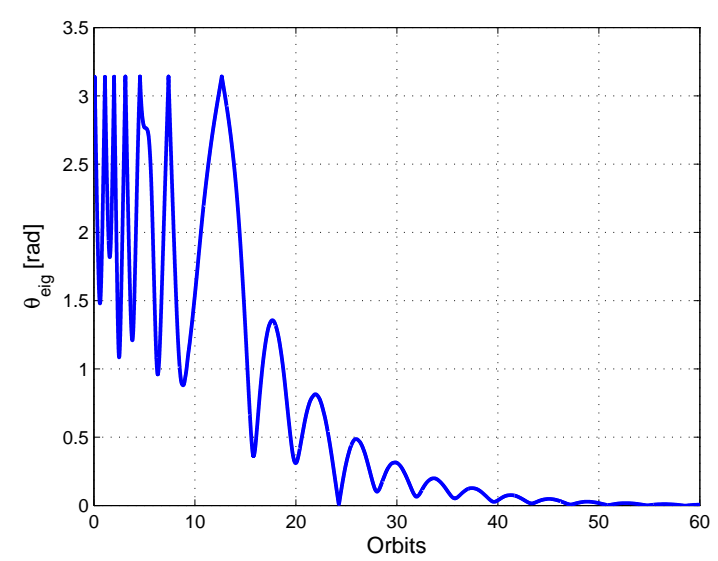

(b) Eigenaxis attitude error for decentralized RCAC

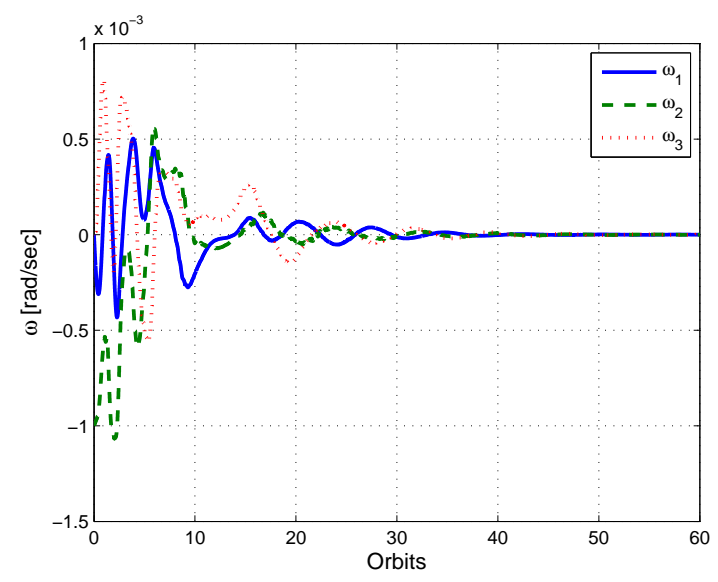

(d) Angular velocity for decentralized RCAC

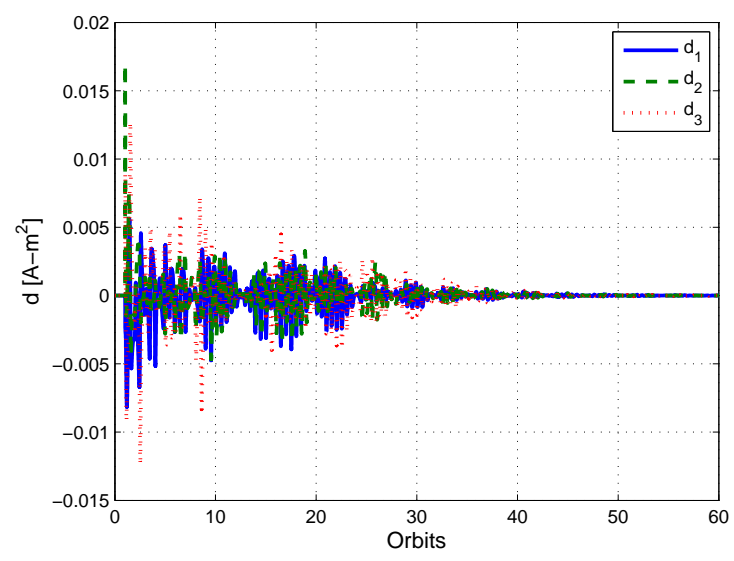

(f) Commanded Dipole for MIMO RCAC

Figure 3. Comparison of RCAC using the average Markov parameter $\tilde{H}$ versus the decentralized approach for the M2R maneuver for the sphere inertia $J_{\text {arbitrary }}$ in (75). 
The advantage of the centralized controller can be attributed to the dimension of the controller parameter $\theta$ and to the information about coupling of the axes in the average input matrix $\tilde{B}_{\mathrm{SC}}$. The loss of information caused by decoupling the input and output relations in (67) increases the settling time of the decentralized architecture in the presence of the real time input matrix $B_{\mathrm{SC}}(t)$.

We can also compare these approaches based on algorithm complexity and execution time. The dimension of the control parameter $\theta$ in (28) affects the memory requirements and time required to compute each control iteration. This is an important factor for the application of these control laws on small spacecraft. For a controller of order $n_{c}$, the averaged Markov parameter method needs to compute $l_{u} n_{c}\left(l_{u}+l_{z}\right)=27 n_{c}$ entries of $\theta$. In contrast, the decentralized approach requires $3 l_{u} n_{c}\left(l_{u}+l_{z}\right)=9 n_{c}$ entries. Thus, the decentralized approach yields similar settling times for M2R maneuvers using a third of the computational cost.

\section{VI.B. M2S Examples}

We command the spacecraft to spin about a body axis aligned in a specific inertial direction. Let the initial angular velocity and attitude of the spacecraft be as in Section VI.A. The desired angular rate

$$
\omega_{d}=0.001\left[\begin{array}{ccc}
0 & -1 & 0
\end{array}\right]^{\mathrm{T}} \mathrm{rad} / \mathrm{sec}
$$

corresponds to a Nadir-pointing attitude. The desired attitude evolves over time according to (6) where the initial desired attitude $R_{d}(0)$ is described by an eigenaxis rotation of $\theta_{d}(0)=96^{\circ}$ about the vector $n_{d}$ in (72). We set the controller parameters as in Table 2. Figures 4 and 5 show that both the MIMO controller with the averaged Markov parameter and the decentralized approach are able to bring the spherical and cylindrical spacecraft into a spin about an inertially pointed body axis. 


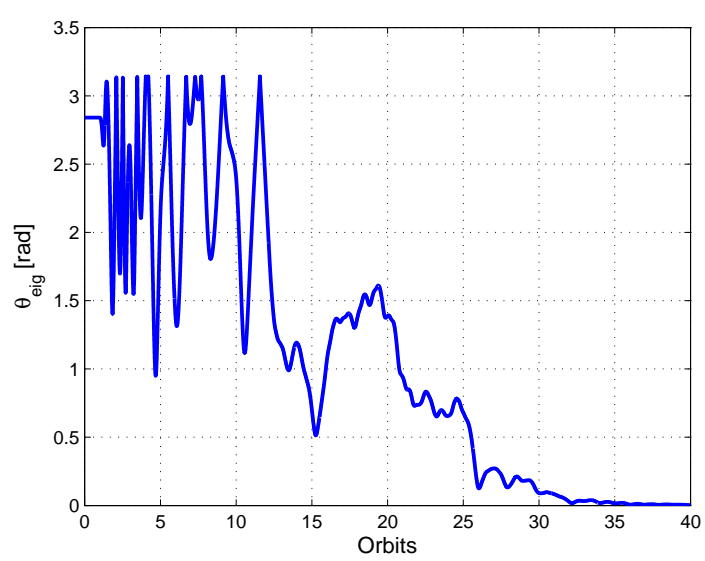

(a) Eigenaxis attitude error for MIMO RCAC

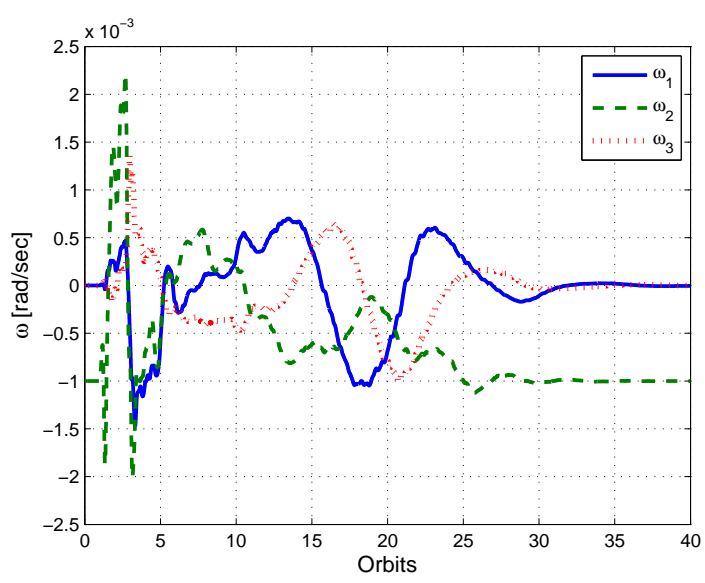

(c) Angular velocity for MIMO RCAC

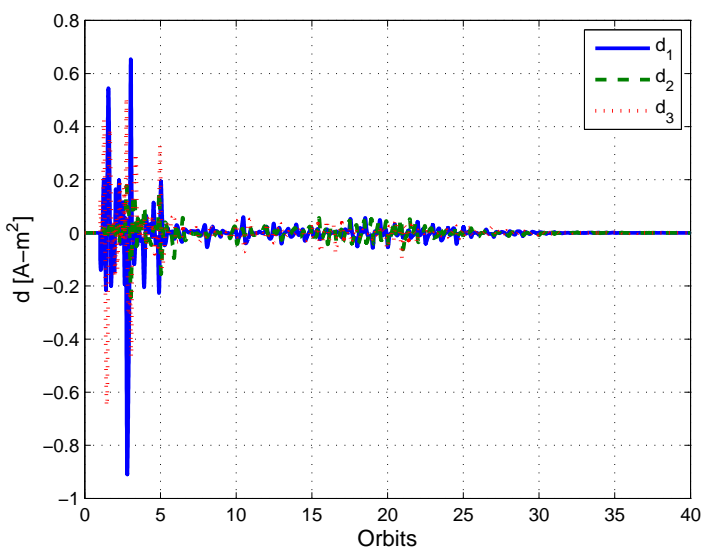

(e) Commanded dipole for MIMO RCAC

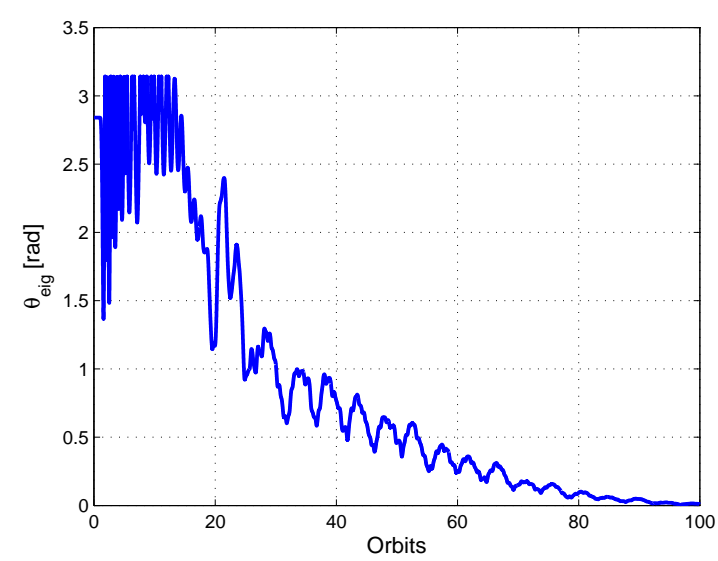

(b) Eigenaxis attitude error for decentralized RCAC

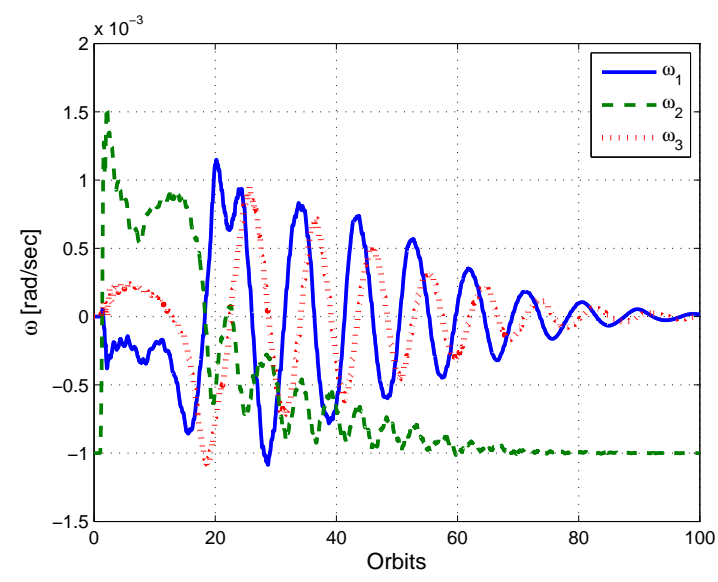

(d) Angular velocity for decentralized RCAC

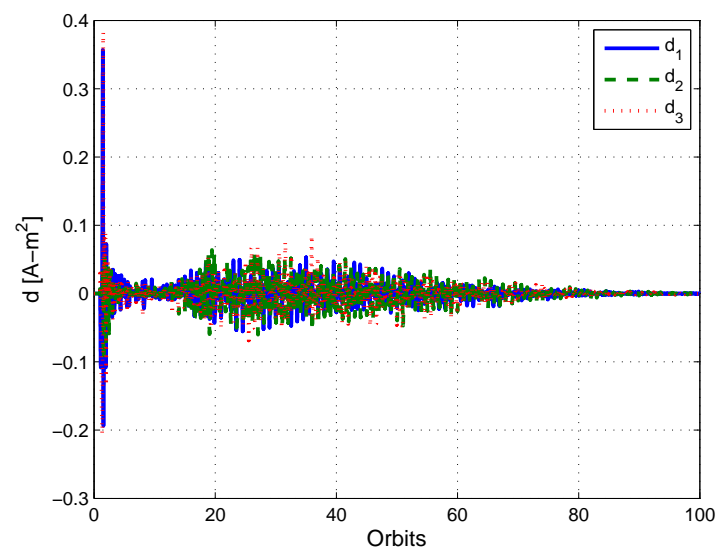

(f) Commanded dipole for decentralized RCAC

Figure 4. Comparison of RCAC using the average Markov parameter $\tilde{H}$ versus the decentralized approach for the M2S maneuver for the sphere inertia $J_{\text {sphere }}$ in (73). 


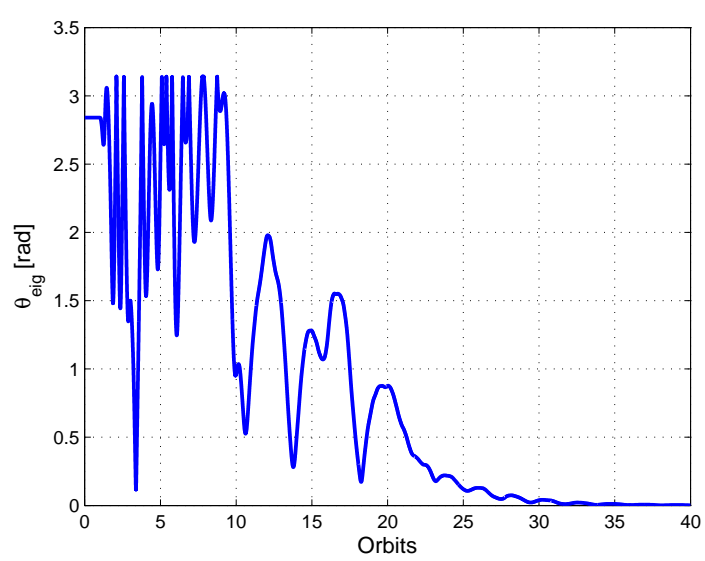

(a) Eigenaxis attitude error for MIMO RCAC

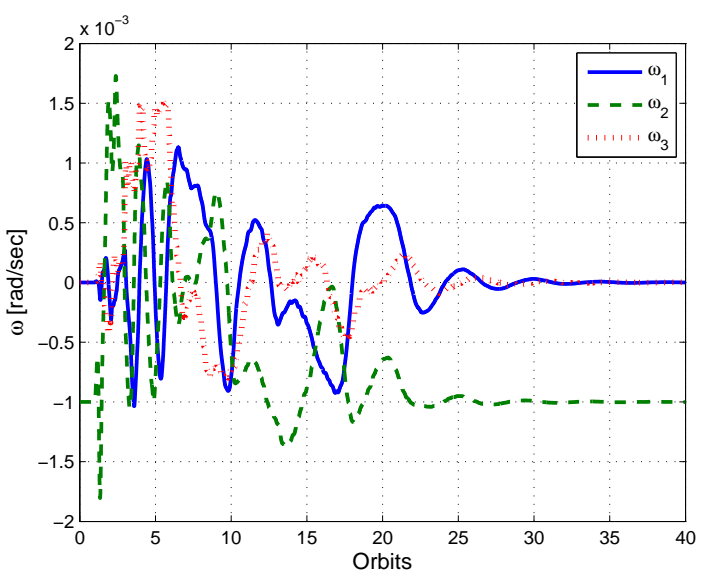

(c) Angular velocity for MIMO RCAC

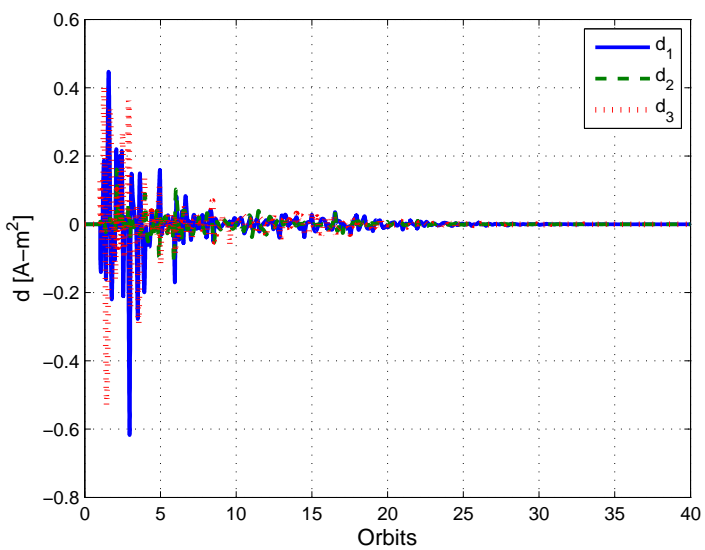

(e) Commanded dipole for MIMO RCAC

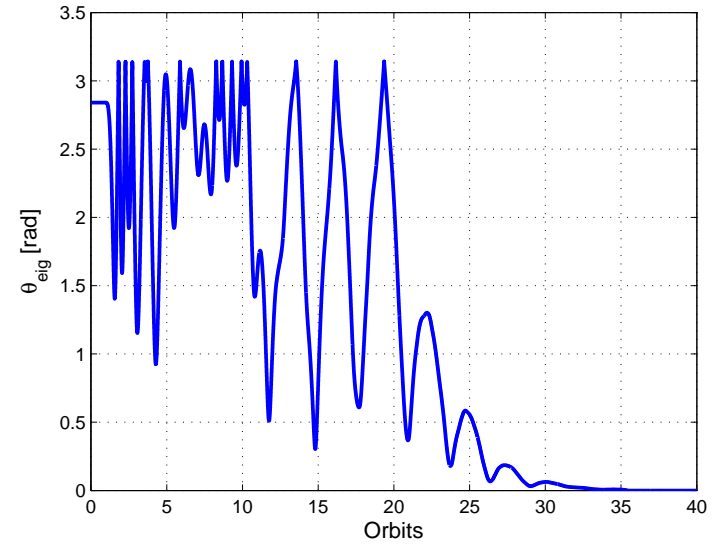

(b) Eigenaxis attitude error for decentralized RCAC

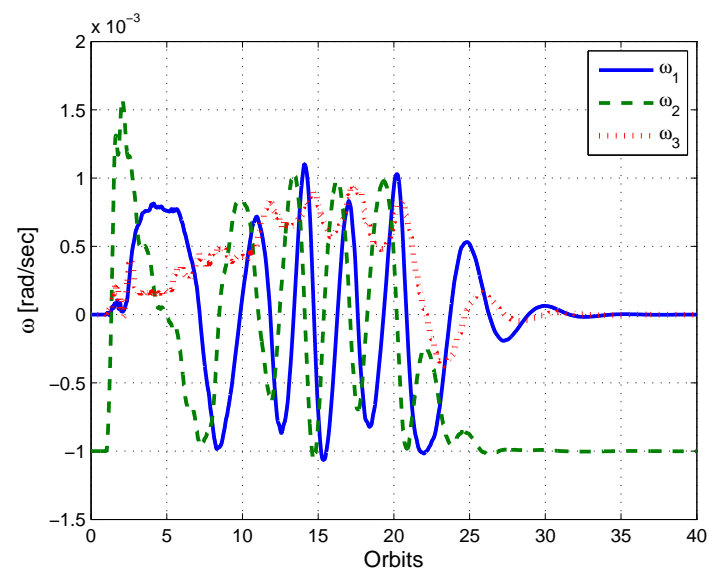

(d) Angular velocity for decentralized RCAC

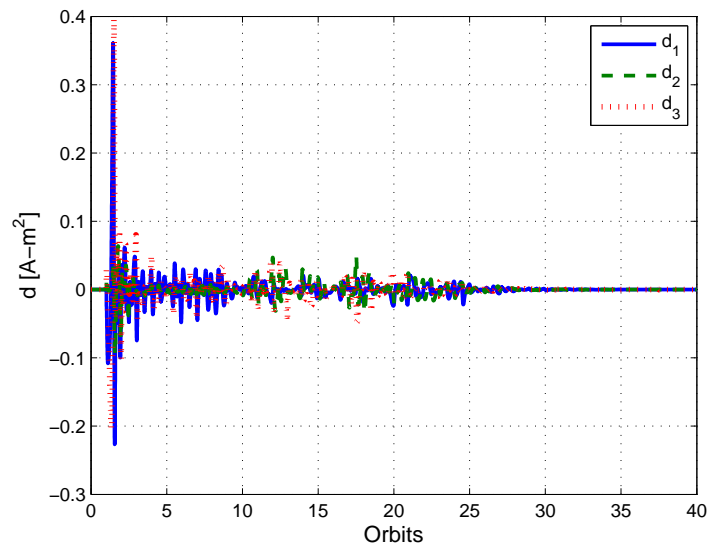

(f) Commanded dipole for decentralized RCAC

Figure 5. Comparison of RCAC using the average Markov parameter $\tilde{H}$ versus the decentralized approach for the M2S maneuver for the cylinder inertia $J_{\text {cylinder in (74). }}$ 


\section{Conclusions and Future Research}

The RCAC algorithm was used to control spacecraft angular rate and attitude using magnetic torque actuators. The torque command computed by RCAC was allocated into magnetic dipoles based on the generalized inverse of the skew symetric cross-product matrix of the magnetic field vector. In order to utilize the RCAC framework, the nonlinear, continuous spacecraft dynamics were linearized to obtain Markov parameters. The Markov parameter obtained was then made left invertible through two different approaches, averaging and decentralized control.

The average of the input matrix used to compute the Markov parameter is positive definite for the high inclination orbits which are of interest for most low-earth-orbit missions. Using the averaged Markov parameter an inertia free stabilizing control law was developed. Numerical simulations show that the algorithm can achieve a M2R maneuver and bring different spacecraft to rest at an inertial attitude.

The decentralized control approach assumes that the sensor and actuator axes are aligned. The three control inputs are computed by independent RCAC controllers using different performance variables. This architecture results in a multi-input, single-output system with left invertible Markov parameters. The decentralized RCAC approach was also shown to complete the M2R maneuver for different inertias.

Comparison of the numerical results indicate that the averaged Markov parameter approach has better settling time characteristics than the decentralized approach given similar RCAC tunings. However, the decentralized method uses one third of the computational capacity of the averaged Markov parameter approach. Thus, the decentralized method is better suitable for applications where computational capacity is limited and settling time requirements are flexible. Furthermore, the settling time of the decentralized approach could be improved by modifying the performance variable to account for the coupling present in the magnetic control formulation.

Future work will focus on extending the implementation of RCAC to use additional Markov parameters. Furthermore, the relation between the performance and controller weighting matrices and the magnitude of the control input will be investigated. A stability analysis of the full closed loop system is also of interest. We also wish to investigate the effects of noise on magnetic field measurement and to address the problem of attitude-only output feedback control using RCAC. The problem of momentum dumping using magnetic torque is also of interest. Finally, we will test the RCAC on a higher fidelity simulator which includes disturbances such as aerodynamic drag, gravity gradient, and solar pressure.

\section{References}

${ }^{1}$ Park, G., Seagraves, S., and McClamroch, N. H., "A Dynamic Model of a Passive Magnetic Attitude Control System for the RAX Nanosatellite," Guidance, Navigation, and Control Conference, AIAA, Toronto, Ontario.

${ }^{2}$ Ismail, Z. and Varatharajoo, R., "A study of reaction wheel configurations for a 3-axis satellite attitude control," Advances in Space Research, Vol. 45, No. 6, 2010, pp. 750-759.

${ }^{3}$ Stickler, A. C. and Alfriend, K. T., "Elementary Magnetic Attitude Control System," Journal of Spacecraft and Rockets, Vol. 13, 1976, pp. 282-287.

${ }^{4}$ Marteru, F., Gabriel, S. R., and Rogers, E., "Attitude determination and control for small spacecraft," Control '96, UKACC International Conference on (Conf. Publ. No. 427), Vol. 1, sept. 1996, pp. 620 - 625 vol.1.

${ }^{5}$ Reyhanoglu, M. and Hervas, J. R., "Three-axis magnetic attitude control algorithms for small satellites," Recent Advances in Space Technologies (RAST), 2011 5th International Conference on, IEEE, 2011, pp. 897-902.

${ }^{6}$ Silani, E. and Lovera, M., "Magnetic spacecraft attitude control: a survey and some new results," Control Engineering Practice, Vol. 13, 2005, pp. 357-371.

${ }^{7}$ Venugopal, R. and Bernstein, D. S., “Adaptive disturbance rejection using ARMARKOV/Toeplitz models,” Transactions on Control Systems Technology, Vol. 8, No. 2, 2000, pp. 257-269.

${ }^{8}$ Santillo, M. A. and Bernstein, D. S., "Adaptive control based on retrospective cost optimization," Journal of guidance, control, and dynamics, Vol. 33, No. 2, 2010, pp. 289-304.

${ }^{9}$ D'Amato, A. M., Sumer, E. D., and Bernstein, D. S., "Frequency-domain stability analysis of retrospective-cost adaptive control for systems with unknown nonminimum-phase zeros," 50th IEEE Conference on Decision and Control and European Control Conference, IEEE, 2011, pp. 1098-1103.

${ }^{10}$ Hoagg, J. B. and Bernstein, D. S., "Retrospective Cost Model Reference Adaptive Control for Nonminimum-Phase Systems," Journal of Guidance Control and Dynamics, Vol. 35, No. 6, 2012, pp. 1767-1786.

${ }^{11}$ Cruz, G., D'Amato, A. M., and Bernstein, D. S., "Retrospective Cost Adaptive Control of Spacecraft Attitude," Guidance, Navigation, and Control Conference, Minneapolis, Minnesota, AIAA.

${ }^{12}$ Cruz, G. and Bernstein, D. S., "Adaptive Spacecraft Attitude Control with Reaction Wheel Actuation," Proceedings American Control Conference, 2013, pp. 4839-4844.

${ }^{13}$ Weiss, A., Cruz, G., Agarwal, K., Rahman, Y., Medikeri, M., Xie, A., Camblor, M., Kolmanovsky, I., and Bernstein, D. S., "Inertia-free Attitude Control Laws Based on Rotation Matrices for Spacecraft with Torquers, Thrusters, and Wheels," Proc. Itzhack Y. Bar-Itzhack Memorial Symposium on Estimation, Navigation, and Spacecraft Control, 2012. 
${ }^{14}$ Sanyal, A., Bernstein, D. S., and Chaturvedi, N., "Inertia-Free Spacecraft Attitude Tracking with Disturbance Rejection and Almost Global Stabilization," Journal of Guidance Control and Dynamics, Vol. 32, 2009, pp. 1167-1178.

${ }^{15}$ Lovera, M. and Astolfi, A., "Global Magnetic Attitude Control of Inertially Pointing Spacecraft," Journal of Guidance Control and Dynamics, Vol. 28, 2005, pp. 1065-1072.

${ }^{16}$ Bayat, F., Bolandi, H., and Jalali, A. A., "A heuristic design method for attitude stabilization of magnetic actuated satellites," Acta Astronautica, Vol. 65, No. 1112, 2009, pp. $1813-1825$.

${ }^{17}$ Finlay, C. C., Maus, S., Beggan, C. D., Bondar, T. N., Chambodut, A., Chernova, T. A., Chulliat, A., Golovkov, V. P., Hamilton, B., Hamoudi, M., et al., "International geomagnetic reference field: the eleventh generation," Geophysical Journal International, Vol. 183, No. 3, 2010, pp. 1216-1230. 